\title{
Contents, Vol. 88, 1936
}

\section{Inhaltsverzeichnis.}

Eigenarbeiten.

Seite

Anargyros, EL, Spontane Zysten der Irishinterfläche . . 93 Biró, I., Über einen Fall vonbeiderseitigem Naevus flammeus

und Glaukom 80

Blaskovics, L. v., Über Zyklektomie 75

Busacca, Arch., Die Entfernung des Tarsalteiles des Orbikularmuskels des Oberlides zwecks Bekämpfung des Entropiums und der Trichiasis zur Verhinderung von Rückfalien des trachomatösen Prozesses 100 Casanovas, /., Über einen Fall von metastatischem Uvealsarkom 86

Fazakas, Alexander, Heilung einer Epithelwucherung in der vorderen Kammer durch Dampfkauterisation .... 315 Fischer, Franz, Zur Differentialdiagnose Pseudoneuritis-

Stauungspapille, Neuritis optica .303

Heine, L., Die Kreuzung der Nervenbahnen und die bilaterale

Symmetrie 7

Hoehne, H., Zur Ätiologie der Makulakolobome 297

Kraupa, Ernst, Zur Frage der , hinteren ringförmigen Glas-

körperabhebung" 224

Müller, H. K., Pupillotonie uud Adiesche Krankheit . . 20 Purtscher, Ernst, Eigenartiger Tumor der Papilla nervi

optici 217

Rintelen, F. , Fundus veränderungen bei tuberöser Hirnsklerose 15 Salzmann, Maximilian, Über Frühstadien des Sarkoms der

Aderhaut und die histologischen Grundlagen des Augen-

spiegelbildes 185

Towbin, B. G., Ein Versuch der Carotinverwendung bei Horn-

hautverpflanzung 107

Trantasj Über die Velonoskiaskopie 230

Vogt, Alfred, Canalis hyaloideus mit Area Martegiani und symmetrisches Foramen hyaloideae posterius. (Hierzu

Tafel I) $\quad 1$

Aus der Praxis für die Praxis.

Kraupa, Ernst, Die Lastexaugenbinde

- IV

Berichte über die ophthalmologische Literatur. Seite 
Aderhaut, Netzhaut (Berichtsjahr vom i. Januar 1934 bis 31. Dezember 1934). Von Prof. W. Riehm-Gießen . . 30

Entwicklungsgeschichte des Auges, Mißbildungen, Anthro-

pologie (Berichtsjahr 1934). Von R. Seef elder-Innsbruck 112

Geschichte der Augenheilkunde. Von R. Seefelder-lnnsb uuck 128

Normale und pathologische Anatomie (Berichtsjahr 1934).

Von Priv.-Doz. Dr. Rudolf Schneider-Qi $\cdot$ à,z

Physiologische und psychologische Optik. Von Dr. Rudolf

Stieber-Innsh $\tau$ vick 248

Vererbung und Auge (Berichtsjahr vom 1. Oktober 1934

bis 1. Oktober 1935). Von A. Francescheíti-Genî . . 319

Physikalische Optik, Dioptrik des Auges, Brillenkunde, Untersuchungsmethoden zur

Refraktionsbestimmung(Berichtsjahr vom 1. Juli 1934 bis 30. Juni 1935). VonDoz. Dr. Rich.

KrämerSRien 328

Gesellschaftsberichte.

Verein Rheinisch-Westfälischer Augenärzte. Sitzung vom 5. Mai

1935 in Düsseldorf 60

Bericht über die zehnte Tagung der Bayerischen Augenärztlichen Vereinigung in München am 23. Juni 1935. Erstattet von

Prof. Dr. A. Passow-München 146

Ophthalmologische Gesellschaft in Wien:

Sitzung vom 21. Oktober 1935166

Sitzung vom 18. Novbr. 1935281

Sitzung vom 16. Dezbr. 1935341

Ungarische ophthalmologische Gesellschaft. Sitzg. vom 6. April 1935170

Sitzungen vom 29. und 30. Mai 1935 285Verhandlungen der Schwedischen Gesellschaft der Augenärzte1934/35. Redigiert von K. O. Granström. Sitzung vom24. November $1934 \quad$ I73

Diagnose und Therapie 62,177, 291, 346

Buchbesprechungen 180, 295, 348

Personalien 296

Hermann Wilbrand $\uparrow(1851-1935)$

Richard Deutschmann $\dagger \quad 74$

Deutsche Ophthalmologische Gesellschaft (Voranzeige) 296

S. Sachregister zu Band $88 \quad 349$

Namenregister zu Band $88 \quad 352$ 